\title{
VALORACIÓN DEL PROFESORADO DE EDUCACIÓN SECUNDARIA DE LA CIUDAD DE TETUÁN SOBRE LA FORMACIÓN EN TIC DESARROLLADA DESDE EL MINISTERIO DE EDUCACIÓN NACIONAL ASSESSMENT OF SECONDARY EDUCATION TEACHERS IN THE CITY OF TETUÁN ABOUT ICT TRAINING DEVELOPED BY THE MINISTRY OF EDUCATION
}

\author{
Dr. Tomás Sola Martínez \\ tsola@ugr.es \\ Dra. Majda Nniya El Berdai² \\ m.nniya@gmail.com \\ Dr. Antonio Moreno Ortiz ${ }^{3}$ \\ amo@uma.es \\ Dr. José Javier Romero Díaz de la Guardia ${ }^{4}$ \\ josejavier.romero@unir.net
(1) Universidad de Granada. Facultad de Ciencias de la Educación. Departamento de Didáctica y Organi- zación Escolar. Campus de Cartuja s/n, 18071, Granada (España).
(2) Reino de Marruecos. Ministerio de Educación Nacional y de la Formación Profesional. Instituto de Enseñanza Secundaria Cualificante "Al Jahed". Cartier Al Adarissa I, 13, (Maroc).
(3) Universidad de Málaga. Facultad de Filosofía y Letras. Departamento de Filología Inglesa, Francesa y Alemana. Campus de Teatinos, 29071, Málaga (España).
(4) Universidad Internacional de la Rioja (UNIR). Facultad de Educación. Departamento de Didáctica y Organización Escolar. Gran Vía Rey Juan Carlos I, 41, 26002, Logroño, La Rioja (España).

Este artículo forma parte de una investigación doctoral que estudia la implantación e integración de las TIC en el sistema educativo de Marruecos. En este estudio pretendemos conocer la valoración del profesorado sobre la fase de implantación de las TIC, a nivel de infraestructura y formación, en los centros de Enseñanza Secundaria de la ciudad de Tetuán. Se emplearon métodos cuantitativos y cualitativos para la recogida y análisis de los datos, concluyendo que la percepción de los encuestados es que la implantación ha sido incompleta. Palabras clave: Investigación educativa, Formación de profesorado, TIC, Educación secundaria.

This article is part of a doctoral research studying the implementation and integration of ICT in the educational system in Morocco. In this study we try to know the teacher evaluation of the ICT implementation phase, at the infrastructure and training level, in secondary schools at the city of Tetuán. Quantitative and qualitative methods for recollection and analysis of data were used, concluding that the perception of the respondents is that the implementation has been incomplete

Keywords: Educational research, Teacher training, ICT, Secondary education.

Palabras clave: Herramienta de aprendizaje, innovación educativa y TIC, entornos de aprendizaje ricos en tecnología, enseñanza.

$$
-49 \text { - }
$$

Píxel-Bit. Revista de Medios y Educación. $N^{o} 50$ Enero 2017. ISSN: 1133-8482. e-ISSN: 2171-7966. doi: http://dx.doi.org/10.12795/pixelbit.2017.i50.03 


\section{Introducción.}

\subsection{Las TIC y la Educación.}

A lo largo de la historia de la educación se han ido dando cambios relevantes que necesariamente acompañan a las revoluciones sociopolíticas y económicas del contexto mundial. Dentro de estos cambios se encuentran las adaptaciones de las políticas educativas en función de la globalización y del ritmo que marca la situación socioeconómica en un país. Es decir, la escuela está sufriendo cambios en los últimos tiempos en torno a las funciones que desempeña según los nuevos modelos de la sociedad, cambios en los roles de los participantes en los procesos de enseñanza y aprendizaje, en las tecnologías de la información y la comunicación (TIC) puestas a disposición de los docentes para desempeñar su labor y cambios en la percepción del contexto formativo en general (Cabero \& Llorente, 2015). Estos cambios en la escuela no están al margen de la influencia tecnológica en la sociedad. A lo largo de años hemos presenciado cómo se van incorporando en las aulas de muchos centros herramientas TIC tales como ordenadores, pizarras digitales, video-proyectores, internet, etc. (Adell \& Castañeda, 2012; Fernández, 2016).

La revolución digital impone fuertes desafíos a la educación obligándola a replantear sus modelos de aprendizaje, procedimientos, estrategias didácticas y recursos, facilitando la integración de las TIC en las aulas mediante la incorporación de nuevas tecnologías y poniendo en práctica metodologías que faciliten el desarrollo de habilidades cognitivas y competencias básicas (Roblizo \& Cózar, 2015) fomentando así una educación de calidad para el alumnado.

Según la Declaración de Incheon en el Foro Mundial sobre la Educación bajo el lema «Educación de calidad, equitativa e inclusiva así como un aprendizaje durante toda la vida para todos en 2030. Transformar vidas mediante la educación»(UNESCO, 2015) entre los aspectos que influyen en la calidad de la educación se encuentra la integración de las TIC en los procesos de enseñanza y aprendizaje, así entre los objetivos que se marcan a nivel internacional para fomentar la calidad de la educación en todos los países del mundo, está la Declaración 10 (UNESCO, 2015):

(...) Nos comprometemos también a fortalecer la ciencia, la tecnología y la innovación. Es preciso aprovechar las tecnologías de la información y la comunicación (TIC) para reforzar los sistemas educativos, la difusión de conocimientos, el acceso a la información, el aprendizaje efectivo y de calidad, y una prestación más eficaz de servicios.

Esta iniciativa de integrar las TIC en la educación no es novedosa puesto que a lo largo de décadas han ido apareciendo cambios en las legislaciones educativas de muchos países para promover su utilización dentro de las prácticas y los procesos de enseñanza y aprendizaje. Las políticas educativas en Europa, América del Norte, Japón y Corea del Sur por ejemplo empezaron a establecer el conocido «modelo 1a1»o «un ordenador por niño», paralelamente se impulsó el proyecto One Laptop per Child, 2011, llevado a cabo por el Massachusetts Institute of Technology (MIT) con el objetivo de proporcionar ordenadores a cada estudiante en las escuelas de los países en desarrollo. En España a su vez, esta política

$$
-50-
$$


del modelo 1:1 se llevó a cabo con el programa Escuela 2.0 durante el periodo 2009-2012 (Area et al., 2014).

Según Valverde, Garrido y Fernández (2010, p.209) «La preparación de los profesores en los usos educativos de la tecnología es un componente clave en todos los planes de reforma educativa y factor clave en el desarrollo de Buenas Prácticas educativas con TIC». Así pues, el indicativo de la formación tecnológica es un requisito imprescindible para la integración de las TIC, según Cabero (2014) es necesario fomentar una adecuada formación inicial y permanente del profesorado mediante la interacción de las diferentes posibilidades que ofrecen las TIC dentro del proceso de enseñanza-aprendizaje.

Entre las competencias mencionadas anteriormente, que se pretenden desarrollar para conseguir una educación de calidad, está la competencia digital. Según Amorós (2013, p.105) «la competencia digital se encuentra entre los aprendizajes esenciales, el concepto de alfabetización se extiende integrando las nuevas tecnologías y la forma razonable de entender las competencias pasa por prestar atención a la formación del profesorado»».

De manera general, la formación del profesorado es la base para lograr una educación de calidad, no sólo antes de comenzar a ejercer la profesión sino también durante la carrera, siendo la formación permanente más importante y la supervisión de la misma permite subsanar la falta de conocimientos, así como mejorar y reforzar las competencias adquiridas (UNESCO, 2009, 2014). Por lo tanto podemos decir que dentro del proceso de enseñanza-aprendizaje las TIC no pueden ir solas sin el docente, «precisamos un profesor abierto al conocimientos y eminenete innovador, capaz de adquirir una mayor autonomía y reposabilidad estratégica, promotor de valores y potenciador de redes de trabajo comunitario» (Trujillo, Aznar \& Cáceres, 2014, p.291). Para ello es necesaria la formación con TIC y sobre las TIC, pero no una formación TIC centrada solo en el aspecto técnico (Llorente, 2008).

Pico (2013, p.69), argumenta que «sin una fundamentación metodológica carece de sentido», es decir, es preciso contar con una formación del profesorado en la que se adquieran nuevas competencias, se necesita desarrollar el pensamiento crítico, compartir el conocimiento, adaptarse a situaciones innovadoras y de cambio en los nuevos contextos educativos y ampliar las capacidades comunicativas y de enseñanzaaprendizaje dentro y fuera del aula. También es preciso seguir trabajando en la integración de las TIC en el currículo escolar de todas las etapas educativas, desde la enseñanza primaria hasta la universitaria.

\subsection{EI Sistema Educativo de Marruecos.}

Siguiendo esta línea de integración, Marruecos tomó la iniciativa de implantar e integrar las TIC dentro de su sistema educativo a través de reformas educativas y proyectos educativos específicos. En 1999 Marruecos creó un marco relevante para las políticas y la articulación de las necesidades y prioridades de la Educación Nacional, La Carta Nacional de Educación y de Formación (CNEF), con el objetivo de desarrollar las competencias que se consideran necesarias para mejorar la calidad de la educación y promover el avance socioeconómico y científico del país. Junto a las competencias básicas que pasan a formar parte del currículo educativo de Marruecos se encuentran las competencias que hacen referencia al

$-51-$

Píxel-Bit. Revista de Medios y Educación. $N^{o} 50$ Enero 2017. ISSN: 1133-8482. e-ISSN: 2171-7966. doi: http://dx.doi.org/10.12795/pixelbit.2017.i50.03 
tratamiento de la información y competencia digital.

La CNEF, como la denominan los profesionales de la política educativa, articula una nueva perspectiva de la educación para el año 2020 y abarca una serie de proyectosguías que se están llevando a cabo desde la fecha de su creación.

En respuesta a una crisis educativa, se creó en el año 2009 el Plan de Urgencia 2009-2012 (Plan d'Urgence 2009-2012) como reforma urgente de todo del sistema educativo marroquí y que llevó consigo muchos programas educativos para mejorar la calidad de educación pública del país (MEN, 2009). Dentro de las bases del Plan de Urgencia y siguiendo lo formulado por la CNEF está la implantación e integración de las TIC en el sistema educativo como un pilar para la mejora de la calidad de la educación marroquí.

\subsection{La implantación e integración de las TIC en el sistema educativo marroquí.}

La implantación e integración de las TIC en las aulas del Reino de Marruecos pasa a ser imprescindible y debe impregnar cada vez más los procesos de enseñanza y aprendizaje, tal y como se refleja en todas las reformas educativas que lleva realizando este país desde 1999 hasta la actualidad. Entre las bases que la CNEF estableció, se encuentra la implantación y la integración de las TIC como parte de la reforma educativa que lleva en ejecución desde el año 2000. Esta implantación se trata en el Área II de la CNEF (APMTE 2009; COSEF, 1999; Maddi, 1999; MEN, 2005a; MEN, 2005b; MEN, 2005c; MEN, 2006; MEN, 2008; MEN, 2011; Royaume du Maroc, 1999).

Para fomentar el uso de las TIC, el artículo 119 de la CNEF nos informa que para optimizar el uso de los recursos educativos y hacer un mayor uso de la tecnología, se hará de las TIC un requerimiento práctico principal en la enseñanza. Las TIC deben ser objeto de uso inmediato, para ello han de llevarse a cabo las siguientes actuaciones: Equipar las aulas con medios tecnológicos y formar a los docentes para afrontar las dificultades vinculadas a la lejanía y el aislamiento de los alumnos destinatarios; Basarse en la educación a distancia en la preparatoria y secundaria, para las zonas rurales; Avanzar hacia la igualdad de oportunidades de acceso a los recursos de información, redes de comunicación y bases de datos resolviendo de forma rápida y barata los problemas relacionados con la distribución inadecuada y desigual de los recursos didácticos básicos.

Según el artículo 120 de la CNEF, se asegurará por parte de todas las instituciones educativas, la adquisición de equipos informáticos y de diversos materiales y herramientas educativas y científicas a través de compras a granel en condiciones preferenciales para los profesores, los alumnos y los administradores. Por último, según el articulo 121 de la CNEF, teniendo en cuenta que la tecnología educativa desempeña un papel importante y creciente en los sistemas y métodos de enseñanza y teniendo en cuenta el artículo 119 de esta Carta, las autoridades educativas y de capacitación se asegurarán de integrar estas tecnologías en la realidad de escuela, basado en el siguiente objetivo: un centro de computación y una biblioteca multimedia en cada escuela al final de la próxima década a partir del año escolar 2000-2001.

El Plan de Urgencia 2009-2012, que surge continuando los pasos de la CNEF, lleva también como objetivos la implantación e integración de las TIC en la educación a través

$$
-52-
$$


de iniciativas como los proyectos E1P8, E1P10 y E3P5 que no abarcaremos aquí por su extensión. Pero sí trataremos uno de los programas que empezó a ejecutarse como prioritarios para la implantación de las TIC en las escuelas y que coincidió con la fase inicial de esta investigación, el programa GENIE.

\subsubsection{El programa GENIE.}

El programa GENIE es el acrónimo de «Generalización de la Estrategia Nacional de Tecnologías de la Información y la Comunicación para la Educación». Iniciada a principios del año 2006, esta estrategia fue estructurada en tres áreas principales (MEN, 2011): Infraestructura: Equipamiento de aulas multimedia conectadas a Internet; La formación del profesorado: conocimientos básicos de informática y el uso de las TIC; y Recursos digitales: la creación de un laboratorio nacional de recursos digitales y de un portal nacional CTBT.

Más adelante el programa GENIE se vuelve a programar durante un período de 5 años (2009-2013) y se refuerza con dos nuevas estrategias: Dirección del programa y desarrollo de los usos de las TIC. Con esta iniciativa, el programa GENIE se vuelve a colocar en el corazón de la reforma de la educación.

Con respecto a la formación del profesorado, en este programa educativo se puso en práctica un esquema de formación «en cascada» en torno a la planificación de las diferentes acciones formativas. Así, un equipo de expertos de Ministerio se encargó de la formación de los inspectores y éstos a su vez tuvieron la responsabilidad de la formar a los equipos directivos y docentes. La formación ha sido estructurada de forma modular, y tras comenzar con una fase de sensibilización e iniciación a las TIC, se han estudiado las aplicaciones educativas de los paquetes ofimáticos y por último se ha profundizado en la integración de las TIC en las distintas disciplinas académicas (MEN, 2011,2015).

\section{Método.}

La metodología (Buendía 1998; Bunge, 1969) hace referencia al metanivel de investigación que aspira a conocer los procesos de investigación. Dependiendo de la concepción que se tenga de la realidad, se formularán los elementos metodológicos y organizativos. Nuestro estudio se enmarca dentro de las investigaciones descriptivas eclécticas, dado que se han utilizado tanto instrumentos de corte cuantitativo como cualitativo, quedando justificada la elección por la propia naturaleza de la investigación.

\subsection{Objetivo.}

El objetivo de nuestra investigación es conocer la valoración de los docentes de los centros educativos de Enseñanza Secundaria de Tetuán sobre la formación que han tenido los profesores sobre las TIC bajo las directrices del Ministerio de Educación Nacional (MEN) de Marruecos.

\subsection{Muestra.}

El problema que planteamos en este estudio va dirigido a la población formada por el profesorado de los centros docentes de educación secundaria de la ciudad de Tetuán, quedando la muestra invitada en cuatro Centros de Enseñanza Secundaria Preparatoria y cuatro Centros de Enseñanza Secundaria Cualificante de Tetuán. El tamaño

$$
-53-
$$

Píxel-Bit. Revista de Medios y Educación. $N^{o} 50$ Enero 2017. ISSN: 1133-8482. e-ISSN: 2171-7966. doi: http://dx.doi.org/10.12795/pixelbit.2017.i50.03 


\begin{tabular}{|ll|r|r|}
\hline & & & \\
\hline Válidos & Hombre & 156 & 66,4 \\
& Mujer & 79 & 33,6 \\
& Total & 235 & 100,0 \\
\hline
\end{tabular}

Tabla 1. Género de los encuestadosFuente: Elaboración propia

de la muestra que ha aceptado participar en este estudio es de 235 sujetos, de los cuales el $66 \%$ son hombres y el $34 \%$ mujeres (Véase Tabla 1).

La edad de la mayoría de los encuestados gira en torno a los 41 y 50 años, dando un porcentaje del $58,3 \%$. El rango de edad siguiente, en un $20,9 \%$, corresponde a docentes de 51 a 60 años. A las edades más jóvenes les corresponden un $19,1 \%$ de 31 a 40 años, y un $1,7 \%$ para los docentes de 21 a 30 años. Por lo que podemos destacar que se trata de una muestra madura y con experiencia en el sector de la docencia.

\subsection{Instrumentos.}

La recogida de datos se ha realizado desde dos tipos de instrumentos, de carácter cuantitativo y de carácter cualitativo según caracteriza a la metodología ecléctica.

\subsubsection{Metodología cuantitativa:} Cuestionario.

La recogida de datos cuantitativos se ha realizado a través de un cuestionario ad-hoc. Para la elaboración del cuestionario se han seguido los pasos de calidad del Método Delphi, por lo tanto, el primer cuestionario acabado fue sometido a Juicio de Expertos con dos miembros de la Universidad de Málaga y cuatro miembros de la Universidad de Granada, ambos pertenecientes a los departamentos de MIDE y Didáctica y Organización Escolar. Tras las modificaciones pertinentes según las recomendaciones de estos expertos, se ha elaborado la versión definitiva del cuestionario para presentar a la muestra, compuesto por un total de 72 ítems y con una escala de acuerdo-desacuerdo tipo Likert, admitiendo cinco posibles valores: (1: totalmente en desacuerdo, 2: en desacuerdo, 3: Ni de acuerdo ni en desacuerdo, 4: de acuerdo, 5: totalmente de acuerdo).

En cuanto a la fiabilidad de nuestro cuestionario, utilizamos el modelo propuesto

\begin{tabular}{|c|c|c|}
\hline $\begin{array}{c}\text { Alfa de } \\
\text { Cronbach }\end{array}$ & $\begin{array}{c}\text { Alfa de } \\
\text { Cronbach } \\
\text { basada en } \\
\text { los } \\
\text { elementos } \\
\text { tipificados }\end{array}$ & $\begin{array}{c}\mathrm{N} \text { de } \\
\text { elementos }\end{array}$ \\
\hline ,962 & 963 & 72 \\
\hline
\end{tabular}

Tabla 2. Estadísticos de fiabilidad.

Fuente: Elaboración propia

por Cronbach en 1951, el estudio de fiabilidad se ha realizado con las 72 variables de carácter cuantitativo que pedían valoraciones de 1 a 5 , según el grado de acuerdo con la afirmación, obteniéndose el siguiente resultado (Véase Tabla 2).

A la luz de estos resultados, podemos afirmar que la fiabilidad del cuestionario orientado al profesorado puede ser calificada de excelente, ya que hemos obtenido un coeficiente Alfa de Cronbach por encima incluso del 0,95.

\subsubsection{Metodología Cualitativa: Grupos de Discusión.}

Con el objeto de profundizar en los datos aportados por el profesorado mediante la aplicación de cuestionario y remediar los posibles sesgos que como técnica de recogida de información pueda poseer el este tipo de

$$
-54-
$$




\begin{tabular}{|l|c|c|c|c|}
\hline Ítems & $\mathbf{N}$ & Media & Desv. típ. & Varianza \\
\hline B12. En los últimos dos años he recibido formación específica en TIC & 235 & 2,25 & 1,337 & 1,787 \\
\hline $\begin{array}{l}\text { B13. El tiempo dedicado a la formación ha sido suficiente para adquirir } \\
\text { conocimientos útiles para mi trabajo. }\end{array}$ & 235 & 1,94 & 1,188 & 1,411 \\
\hline $\begin{array}{l}\text { B14. La formación que he recibido en TIC y en sus posibilidades como } \\
\text { herramientas educativas han respondido a mis necesidades de formación }\end{array}$ & 235 & 2,09 & 1,266 & 1,603 \\
\hline $\begin{array}{l}\text { B15. Considero que la formación recibida en el uso específico de las TIC } \\
\text { con fines educativos me ha sido útil para mis prácticas docentes y } \\
\text { administrativas }\end{array}$ & 235 & 2,20 & 1,311 & 1,719 \\
\hline $\begin{array}{l}\text { B16. Actualmente tenemos una buena oferta de formación disponible para } \\
\text { aprender a utilizar las TIC en los procesos de enseñanza-aprendizaje. }\end{array}$ & 235 & 2,02 & 1,261 & 1,589 \\
\hline
\end{tabular}

Tabla 3. Estadísticos descriptivos. Fuente: elaboración propia.

instrumento, se planteó recoger datos cualitativos mediante la técnica del grupos de discusión. Para el diseño del grupo de discusión se tuvo en cuenta la diversidad profesional de los participantes, el medio o espacio en que se va a desarrollar el grupo de discusión, la herramienta con la cual se llevará a cabo el registro de los datos obtenidos por este instrumento y el tiempo que va durar la discusión. Tal y como señala Colás (1999, 2001), Barbour y Kitzinger (1999), Canales y Peinado (1994) Frey y Fontana (1993), la técnica del grupo de discusión permite analizar discursos de un número de personas, que representan a la comunidad que se estudia, reunidas con el fin de mantener una discusión moderada por un tercero, el cual solo es un observador que dirige en caso necesario el discurso.En esta investigación se ha procedido a la elaboración de cuatro grupos de discusión, dos pertenecientes a los centros de Enseñanza Secundaria Preparatoria y dos centros de Enseñanza Secundaria Cualificante de la ciudad de Tetuán.

\subsection{Procedimientos y estrategias de análisis de datos.}

Para llevar a cabo el análisis de datos cuantitativos se ha utilizado el Paquete Estadístico IBM SPSS Statistics, versión 20.0 para Windows. El proceso consistió en importar todos los datos, etiquetarlos y realizar el análisis estadístico. En relación a los datos cualitativos extraídos de los grupos de discusión, se procedió a una categorización manual a través de la elaboración de un registro y un sistema de codificación mediante el cual se van combinando por asociación semántica diferentes categorías que a su vez forman parte de otras estructuras de significado mayor: las metacategorías. A continuación se procedió a la triangulación de datos como estrategia metodológica para conseguir una mayor comprensión de la realidad estudiada. Según Aguilar y Barroso (2015, p.79) «combinar los diferentes modos de abordar un mismo fenómeno nos va a permitir llegar a conclusiones más profundas y rigurosas sobre el mismo».

\section{Resultados.}

\subsection{Análisis Cuantitativo.}

\subsubsection{Resultados descriptivos.}

Teniendo en cuenta el objetivo de investigación y el contexto del estudio, presentamos la Tabla 3 resumen tras la aplicación de estadísticos descriptivos (media, varianza y desviación típica) al conjunto de ítems del cuestionario sobre la

$-55-$

Píxel-Bit. Revista de Medios y Educación. $N^{o} 50$ Enero 2017. ISSN: 1133-8482. e-ISSN: 2171-7966. doi: http://dx.doi.org/10.12795/pixelbit.2017.i50.03 


\begin{tabular}{|l|c|c|}
\hline \multicolumn{1}{|c|}{ Válido } & Frecuencia & Porcentaje \\
\hline Totalmente en Desacuerdo & 115 & 48,9 \\
\hline En Desacuerdo & 52 & 22,1 \\
\hline Ni de Acuerdo ni en Desacuerdo & 15 & 14,9 \\
\hline De Acuerdo & 15 & 6,4 \\
\hline Totalmente de Acuerdo & 18 & 7,7 \\
\hline Total & 235 & 100,0 \\
\hline
\end{tabular}

Tabla 4. Valoración docente sobre la actual oferta formativa TIC. Fuente: elaboración propia

valoración de la formación en TIC como parte del proceso de implantación del proyecto GENIE.

Según las bases del proyecto GENIE (MEN 2015, 2011), se han ido organizando actividades formativas en TIC para los docentes, directivos e inspectores. Respecto al ítem sobre la formación recibida en los últimos dos años, el $42.1 \%$ del equipo educativo de los centros manifiestan estar en total desacuerdo con el ítem. El 19.6\% afirma estar en desacuerdo, frente al $8.5 \%$ que está en total acuerdo y el $11.9 \%$ de acuerdo. Se mantiene un porcentaje del $17.9 \%$ que no emiten un juicio claro. Los datos reflejan que no se considera suficiente la duración de la formación obtenida en un $71.9 \%$, mientras que el $11.5 \%$, sí la consideran suficiente para adquirir conocimientos útiles para el trabajo docente. El resto mantiene una respuesta neutra. En cuanto al tiempo, la mayoría de los encuestados están en total desacuerdo con la afirmación, ya que no consideran que el tiempo dedicado a la formación sea el suficiente para adquirir conocimientos TIC útiles para su trabajo. En cuanto a la utilidad de la formación recibida en TIC para la práctica docente y administrativa, el $65.1 \%$ no la considera útil frente al $20 \%$, que sí considera útil para su trabajo la formación recibida para el uso especifico de las TIC con fines educativos. El 14.9\% restante no muestra una posición definida respecto al ítem. Como podemos apreciar en la Tabla 4 y Gráfico 1, el



Gráfico 1. Valoración de los docentes sobre la realización de formación TIC en los últimos dos años. Fuente: Elaboración propia.

$$
-56-
$$




\begin{tabular}{|l|r|r|r|}
\hline & Valor & gl & \multicolumn{2}{|c|}{$\begin{array}{c}\text { Sig. asintótica } \\
\text { (bilateral) }\end{array}$} \\
\hline Chi-cuadrado de Pearson & $26,163^{a}$ & 12 &, 010 \\
Razón de verosimilitud & 24,435 & 12 &, 018 \\
Asociación lineal por & 1,276 & 1 &, 259 \\
lineal & 235 & & \\
N de casos válidos & & & \\
\hline
\end{tabular}

a. 9 casillas $(45,0 \%)$ tienen una frecuencia esperada inferior a 5 . La frecuencia mínima esperada es ,26.

Tabla 5. Prueba de $\div 2$-Edad * Actualmente tenemos una buena oferta de formación disponible para aprender a utilizar las TIC en los procesos de enseñanza-aprendizaje.

Fuente: elaboración propia

$48,9 \%$ y el $22,1 \%$ está en total desacuerdo o en desacuerdo con que el Gobierno esté ofreciendo, en el momento de presentar los cuestionarios, una buena oferta formativa en aplicación de las TIC en los procesos de enseñanza-aprendizaje.

\subsubsection{Análisis de Contingencias.}

En esta parte del estudio perteneciente al análisis cuantitativo, pasamos a mostrar tablas de contingencia que representan los diferentes cruces entre algunas variables utilizadas en nuestra investigación. Siguiendo a autores como Moore (2005), que incluyen este tipo de análisis en sus investigaciones, se han determinado si las variaciones de las proporciones de las variables de la tabla de contingencia se deben a que existe una verdadera dependencia entre ellas o a causas aleatorias. En el caso del estudio de tablas de contingencia, la prueba realizada es la prueba elaborada por Pearson (1900, citado en Moore, 2005) denominada Chi-Cuadrado o Ji Cuadrado $(\div 2)$.

Como observamos en la Tabla 5 , se ha podido encontrar que existe relación entre la edad y la variable que hace referencia a la valoración de la oferta formativa. Siendo la significación asintótica: 0.010 menor que alfa: 0.05 se rechaza la hipótesis nula y se concluye que sí existen diferencias estadísticamente significativas entre la variable edad y la variable «actualmente tenemos una buena

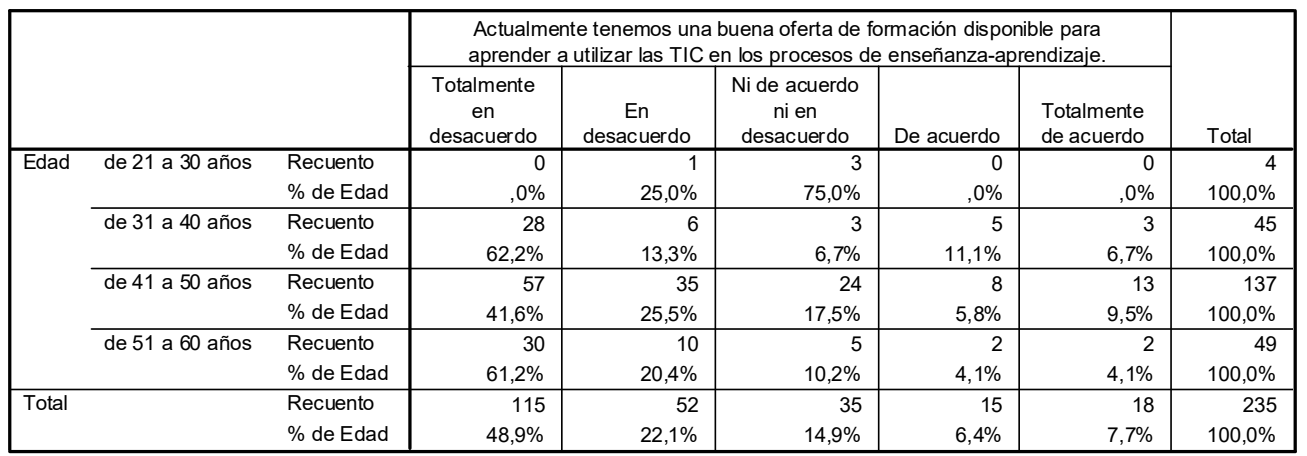

Tabla 6. Tabla de contingencia Edad * Actualmente tenemos una buena oferta de formación disponible para aprender a utilizar las TIC en los procesos de enseñanzaaprendizaje. Fuente: elaboración propia

$$
-57-
$$

Píxel-Bit. Revista de Medios y Educación. No 50 Enero 2017. ISSN: 1133-8482. e-ISSN: 2171-7966. doi: http://dx.doi.org/10.12795/pixelbit.2017.i50.03 


\begin{tabular}{|c|c|c|}
\hline \multicolumn{2}{|c|}{$\begin{array}{l}\text { Medida de adecuación muestral de } \\
\text { Kaiser-Meyer-Olkin. }\end{array}$} & 897, \\
\hline \multirow[t]{3}{*}{$\begin{array}{l}\text { Prueba de esfericidad } \\
\text { de Bartlett }\end{array}$} & $\begin{array}{l}\text { Chi-cuadrado } \\
\text { aproximado }\end{array}$ & 12558,536 \\
\hline & $\mathrm{gl}$ & 2556 \\
\hline & Sig. & ,000 \\
\hline
\end{tabular}

Tabla 7. KMO y prueba de Bartlet. Fuente: elaboración propia

oferta de formación disponible para aprender a utilizar las TIC en los procesos de enseñanza-aprendizaje» con un nivel de confianza del $95 \%$.

Para ver en mayor detalle las diferencias encontradas, en la siguiente tabla de contingencia (Tabla 6) podemos observar que el porcentaje de la valoración «totalmente en desacuerdo» sigue siendo el más alto, y de una forma más específica los docentes con edades entre 31 y 40 años son los que tienen mayor porcentaje en esta respuesta con un $62,2 \%$, muy cercana a los docentes de 51 a 60 años con $61,2 \%$ y de 41 a 50 años con el $41,6 \%$. Los docentes encuestados de menor edad no manifiestan una posición tan clara al respecto, y tienen una mejor valoración de la actual oferta formativa.Tabla 6. Tabla de contingencia Edad* Actualmente tenemos una buena oferta de formación disponible para aprender a utilizar las TIC en los procesos de enseñanza-aprendizaje. Fuente: elaboración propia

\begin{tabular}{|l|l|l|}
\hline Ítem & \multicolumn{1}{|c|}{ Inicial } & Extracción \\
\hline B12 & 1,000 &, 686 \\
\hline B13 & 1,000 &, 728 \\
\hline B14 & 1,000 &, 792 \\
\hline B15 & 1,000 &, 707 \\
\hline B16 & 1,000 &, 708 \\
\hline
\end{tabular}

Tabla 8. Comunalidades. Fuente: elaboración propia

\subsubsection{Análisis Factorial.}

Hemos procedido al análisis de los componentes principales de los datos generales de esta investigación. Seleccionando las variables pertinentes para la elaboración de este artículo obtenemos los siguientes resultados:

Observamos que el requisito de que existan unas correlaciones suficientes entre las distintas variables objeto de estudio queda satisfecho según indica la prueba de esfericidad de Bartlett (Tabla 7). La prueba KMO muestra un valor de 0,897 , lo que podemos calificar de excelente para aplicar el análisis. Siguiendo el proceso del Análisis Factorial, a partir de la matriz de correlaciones se generan una serie de indicadores que nos deben conducir a resolver dos primeras cuestiones: en primer lugar, si existe alguna variable que deba ser desechada para el análisis factorial; en segundo, determinar cuántos factores tomaremos para realizar el análisis. Para responder a la primera pregunta hemos reducido las variables a través de la tabla 8 de Comunalidades y en nuestro caso no excluiremos ninguna variable ya que los porcentajes explicados son muy elevados, todos presentan Comunalidades superiores a 0.50 lo que las hace ser susceptibles de ser retenidas en el análisis.

Para extraer factores se utilizan diversos métodos pero nosotros recurriremos al método de componentes principales. Una vez

$$
-58-
$$




\begin{tabular}{|c|c|c|c|c|c|c|c|c|c|c|c|c|c|c|}
\hline & \multicolumn{14}{|c|}{ Componente } \\
\hline & 1 & 2 & 3 & 4 & 5 & 6 & 7 & 8 & 9 & 10 & 11 & 12 & 13 & 14 \\
\hline B12 & ,491 &,- 109 & ,201 & ,262 & ,206 &,- 443 & ,094 & ,150 & ,017 &,- 128 &, 070 &,- 074 &, 164 &,- 024 \\
\hline B13 & ,496 &,- 459 &, 113 & ,054 & ,258 &,- 301 & ,066 & ,138 & ,122 &,- 210 & ,098 &,- 051 & 039 &,- 045 \\
\hline B14 & ,537 &,- 423 & , 140 & ,116 & ,302 &,- 313 & ,147 & ,057 & ,162 &,- 150 & ,068 & ,057 & 012 &,- 144 \\
\hline B15 & ,462 &,- 164 & ,237 & , 169 & 315 &,- 376 & , 178 & ,077 & ,196 & , 120 & ,018 &,- 090 & 060 &,- 194 \\
\hline B16 & ,583 &,- 418 &, 013 & ,091 & ,314 &,- 159 & ,033 & ,203 & ,055 &,- 055 & ,045 & ,056 &,- 045 &,- 075 \\
\hline
\end{tabular}

Tabla 9. Análisis de Componentes Principales-Adaptado. Fuente: elaboración propia

determinado que el número de factores serán catorce para este estudio, el paso siguiente es asignar puntuaciones a cada una de las variables, con el objeto de determinar las agrupaciones factoriales. En este sentido, la tabla de solución factorial contiene las correlaciones entre cada uno de los factores y cada una de las variables originales llamadas pesos, cargas o saturaciones varían entre -1 y 1. A partir de esta matriz (Tabla 9) ya se podría comenzar a especular sobre las variables que quedarían englobadas dentro de cada factor, que serán las que presenten una mayor saturación para dicho factor. Sin embargo, se recurre a la rotación cuyo objetivo es conseguir que los factores sean lo más interpretables posibles. Esto se consigue aproximando cada factor a un conjunto de variables empíricas y alejándolo lo más posible de otras variables empíricas, de esta forma cada factor explica dimensiones diferentes a los otros factores. Una matriz es tanto más interpretable cuando más se acerque a la Estructura Simple de Thurstone. Esta estructura se basa fundamentalmente en tres criterios: Cada variable empírica está muy saturada en un factor y poco saturada en otros; Cada factor tiene muy pocas cargas significativas; La distribución de cargas de cada factor es diferente a los otros factores.

Método de extracción: Análisis de componentes principales a 14 componentes extraídos.
En nuestro caso se ha realizado la rotación ortogonal mediante el método Varimax para determinar una nueva tabla donde los componentes rotados permitan facilitar la interpretación de la solución factorial.

Método de extracción: Análisis de componentes principales. Método de rotación: Normalización Varimax con Kaiser.a La rotación ha convergido en 17 iteraciones.

A partir de las consideraciones de Thurstone sobre la tabla de componentes rotados (tabla 10), sacamos el factor que corresponde al estudio de este artículo y es el Factor 6. Dentro de este factor hemos de incluir las siguientes variables: B12 - En los últimos dos años he recibido formación específica en TIC.; B13 - El tiempo dedicado a la formación ha sido suficiente para adquirir conocimientos útiles para mi trabajo.; B14 La formación que he recibido en TIC y en sus posibilidades como herramientas educativas han respondido a mis necesidades de formación.; B15 - Considero que la formación recibida en el uso específico de las TIC con fines educativos me ha sido útil para mis prácticas docentes y administrativas.; B16 Actualmente tenemos una buena oferta de formación disponible para aprender a utilizar las TIC en los procesos de enseñanzaaprendizaje. Por tanto, podemos denominar a este Factor 6 «Formación docente en TIC».

$$
-59 \text { - }
$$

Píxel-Bit. Revista de Medios y Educación. $N^{o} 50$ Enero 2017. ISSN: 1133-8482. e-ISSN: 2171-7966. doi: http://dx.doi.org/10.12795/pixelbit.2017.i50.03 


\begin{tabular}{|c|c|c|c|c|c|c|c|c|c|c|c|c|c|c|}
\cline { 2 - 17 } \multicolumn{1}{c|}{} & \multicolumn{10}{c|}{ Componente } \\
\cline { 2 - 16 } & $\mathbf{1}$ & $\mathbf{2}$ & $\mathbf{3}$ & $\mathbf{4}$ & $\mathbf{5}$ & $\mathbf{6}$ & $\mathbf{7}$ & $\mathbf{8}$ & $\mathbf{9}$ & $\mathbf{1 0}$ & $\mathbf{1 1}$ & $\mathbf{1 2}$ & $\mathbf{1 3}$ & $\mathbf{1 4}$ \\
\hline B12 &, 195 &, 042 &, 178 &, 089 &, 132 &, 726 &, 089 &, 195 &,- 047 &,- 014 &, 025 &, 022 &, 059 &,- 097 \\
\hline B13 &,- 043 &, 304 &, 049 &, 104 &, 265 &, 722 &, 031 &, 015 &, 057 &,- 094 &, 081 &, 067 &, 058 &, 021 \\
\hline B14 &, 030 &, 278 &, 055 &, 119 &, 268 &, 755 &,- 047 &, 068 &, 175 &, 009 &, 106 &, 038 &,- 032 &, 050 \\
\hline B15 &, 235 &, 164 &, 011 &, 082 &, 136 &, 715 &,- 036 &, 146 &, 078 &, 177 &,- 148 &,- 047 &,- 040 &, 045 \\
\hline B16 &,- 014 &, 428 &, 177 &, 028 &, 245 &, 605 &, 105 &, 029 &, 180 &, 065 &, 076 &, 085 &, 037 &, 051 \\
\hline
\end{tabular}

Tabla 10. Matriz de componentes rotados - adaptada. Fuente: elaboración propia.

\subsection{Análisis cualitativo.}

Se trata de una categorización manual de la información transcrita de los cuatro grupos de discusión realizados, en base a las «metacategorías»y «categorías» a las que se refieren los objetivos de nuestra investigación. De estas categorías, seleccionamos para el objeto de estudio que concierne este artículo la Categoría 2.2. Duración y contenido de la formación TIC. Tras el análisis de contenido, las respuestas del grupo de discusión 1 las sintetizamos en los siguientes puntos en función del sentido de la respuesta. Hablando de la formación en general: No hubo prácticas durante la formación; Los contenidos no se ajustaban al tiempo debido al bajo nivel que tenían los docentes y al horario laboral que coincidía con la formación Tratando el tema de los contenidos que se llevaron a cabo en la formación: Los contenidos eran rigurosos y adecuados; Los contenidos consistían en multimedia y office (Word y Excel).

Sintetizamos las respuestas obtenidas por el grupo de discusión 2: la formación no era suficiente y consideran adecuado repetirla, a su vez dicha formación no se completó por causa del tiempo. Consideran que era poco tiempo para la cantidad de contenidos a tratar. En relación a los contenidos, los estiman adecuados pero no coincidían con el nivel de los formantes. Por último, consideran que la formación fue más teórica que práctica y que el espacio en el que desarrolló la misma no era adecuado.

Entre los miembros del tercer grupo de discusión encontramos a un formador «facilitador», que aportó una visión más positiva de las acciones formativas llevadas a cabo, indicándonos que la formación cumplió con los objetivos marcados ya que consistía en sensibilizar y motivar a los docentes en TIC y no trabajar directamente con las TIC.

Del grupo de discusión 4 obtenemos lo siguiente: Las valoraciones positivas de la formación: según la opinión de dos participantes del grupo de discusión, la formación fue adecuada para sensibilizar al profesorado en el uso de las TIC y otro participante considera los contenidos adecuados y acordes a lo establecido por el Ministerio, a la vez que adaptados al nivel de los docentes en formación. Las valoraciones negativas de la formación: la opinión mayoritaria de los participantes del estudio nos indica que la formación no fue suficiente si la comparamos con el nivel de los usuarios; no había conexión a Internet; la adquisición de competencias fue limitada; los contenidos no eran suficientes y se dedicaron a la enseñanza del uso del ordenador; los objetivos de la formación no eran claros; y la formación no se culminó en su totalidad. Los obstáculos que provocaron el fracaso de la formación según la dirección del instituto fueron: La temporalización, ya que no se

-60 - 
ajustaba al horario laboral de los usuarios; la falta de conexión a Internet que hizo que s por lo que sólo se formó a los usuarios en Office; y la ausencia de algunos profesores a las sesiones de formación TIC.

\section{Conclusiones.}

Tras llevar a cabo un proceso de triangulación de los datos una vez administrados los instrumentos de investigación, destacamos una serie de conclusiones en torno a la valoración de la formación en TIC por parte del profesorado participante en este estudio.

La formación recibida ha sido considerada como insuficiente en diversos sentidos, en primer lugar porque la temporalización no ha sido adecuada para el cumplimiento de los objetivos establecidos al inicio. Además, se ha destacado la necesidad de mejorar la formación del profesorado respecto a integración de las TIC en la práctica docente, que según el actual programa GENIE está excesivamente centrada en cuestiones de carácter técnico como la multimedia y la ofimática, siendo necesario centrar el hilo conductor de los diferentes cursos en las finalidades educativas de estas tecnologías. Consideramos que es preciso la planificación de itinerarios formativos con diferente nivel de profundización en torno a la utilización e integración de las TIC en el aula, pues se ha constatado que las actividades formativas que abarca el programa GENIE no han cubierto las expectativas de todo el personal docente, y en gran parte de los casos han sido consideradas como una fase de sensibilización y acercamiento inicial a las TIC.

Respecto a la oferta formativa en TIC al alcance de los profesionales de la enseñanza en el contexto de este estudio, cabe señalar una mejor valoración por parte de los docentes más jóvenes, cuestión que puede reflejar una mejor preparación y formación inicial de estos docentes, si bien se ha puesto de manifiesto la necesidad de mejorar la infraestructura tecnológica y de comunicaciones a fin de diseñar escenarios formativos centrados en la adquisición de competencias digitales por parte del profesorado y con una vinculación directa a la práctica docente de los docentes en formación.

\section{Referencias bibliográficas.}

Adell, J. \& Castañeda, L. (2012). Tecnologías emergentes, ¿pedagogías emergentes?. En J. Hernández, M. Pennesi, D. Sobrino y A. Vázquez (Coords), Tendencias emergentes en educación con TIC (pp.13-33). Barcelona: Asociación Espiral, Educación y Tecnología

Aguilar, S. \& Barroso, J. (2015). La triangulación de datos como estrategia en investigación educativa. Píxel-Bit. Revista de Medios y Educación, 45, 73-88. doi: 10.12795/ pixelbit.2015.i47.05

Amorós, L. (2013). Aproximación a la competencia digital. RIDU: Revista d'innovació docent universitaria, 5, 98-109.

APMTE (2009). La Charte. La lettre royale de la rentrée scolaire ,2000-2001; Dahir de 1958 concernant la création d'associations ; La Charte nationale de l'éducation et de la formation, 2008 ; Les textes juridiques et organisationnels en la matière; Les projets $d u$ Plan d'Urgence, 2009. Ministère de l'Education Nationale du Maroc.

Area, M., Alonso, C., Correa, J. M., Del Moral, M. E., De Pablos, J., Peirats, J., ...Valverde, J. (2014). Las políticas educativas TIC en España después del Programa Escuela

$-61-$

Píxel-Bit. Revista de Medios y Educación. $N^{o} 50$ Enero 2017. ISSN: 1133-8482. e-ISSN: 2171-7966. doi: http://dx.doi.org/10.12795/pixelbit.2017.i50.03 
2.0: Las tendencias que emergen. Relatec. Revista Latinoamericana de Tecnología Educativa, 13 (2), 11-33.

Buendía. L. (1998). La investigación por encuesta. En L. Buendía, P. Colás y F. Hernández, Métodos de investigación en Psicopedagogía (pp.119-155). Madrid: McGrawHill

Bunge, M. (1969). La investigación científica. Barcelona: Ariel.

Cabero, J. (2014). Formación del profesorado universitario en TIC. Aplicación del método Delphi para la selección de los contenidos formativos. Educación XX1, 17(1), 111-132. doi: 10.5944/ educxx1.17.1.10707

Cabero, J. \& Llorente, M.C. (2015). Tecnologías de la Información y la comunicación (TIC): escenarios formativos y teorías del aprendizaje. Revista Lasallista de investigación, 12 (2), 186-193.

COSEF (1999). Charte Nationale de l'Education et de la Formation. Royaume $d u$ Maroc. Recuperado de http:// www.alpha.gov.ma

Fernández, L. (2016). El uso didáctico y metodológico de las tabletas digitales en aulas de educación primaria y secundaria de Cataluña. Píxel-Bit. Revista de Medios y Educación, 48, 9-25. doi: 10.12795/ pixelbit.2016.i48.01

Llorente, M. C. (2008). Aspectos fundamentales de la formación del profesorado en TIC. Píxel-Bit. Revista de Medios y Educación, 31, 121-130. Recuperado de http://www.sav.us.es/pixelbit/pixelbit/ articulos/n31/n31 art/art319.htm

Maddi, L. (1999). As siyassa at taalimya bi Al-Maghreb wa rihanat al moustaqbal. Rabat: Faculté de Sciences de 1'Education.

MEN (2005a). Cadre stratégique de développement du système éducatif (2005).
Direction de la stratégie et de la planification. Rabat : Département de l'Éducation National. Ministère de l'Éducation Nationale, de l'Enseignement Supérieur, de la Formation des Cadres et de la Recherche Scientifique.

MEN (2005b). Réforme du système éducatif: Bilan et perspectives. Rabat: Département de l'Éducation National. Ministère de l'Éducation Nationale, de l'Enseignement Supérieur, de la Formation des cadres et de la Recherche Scientifique.

MEN (2005c). Plan National de l'Education pour tous. Rabat: Ministère de l'Éducation Nationale, de l'Enseignement Supérieur, de la Formation des Cadres et de la Recherche Scientifique.

MEN (2006). Généralisation des TIC dans l'Enseignement : Plan d'Action pour l'axe « formation " $d u$ programme GENIE. Programme GENIE.

MEN (2008). Analyses des besoins et des priorités en matière des ressources pédagogiques numériques. Rapport Final. Education et territoires. Programme GENIE.

MEN (2009). Rapport Résumé $d u$ Programme d'Urgence 2009-2012. Rabat: Ministère de l'Éducation Nationale, de l'Enseignement Supérieur, de la Formation des Cadres et de la Recherche Scientifique.

MEN (2011). Généralisation des TIC dans l'Enseignement : Tous pour la réussite de l'intégration des TIC dans l'Enseignement. Programme GENIE.

MEN (2015). Les Mesures Prioritaire pour la Réforme de l'École Marocain 2015-2030. Royaume du Maroc. Recuperado de http:// www.men.gov.ma/sites/fr/default.aspx

Moore, D.S. (2005). Estadística aplicada básica. Barcelona: Antoni Bosch.

Pico, S. (2013). Formación TIC del profesorado para garantizar el éxito en la

$-62-$ 
integración de la tecnología. Itaca: revista de filología, 4, 65-80.

Roblizo, M. J. \& Cózar R. (2015). Uso y competencias en TIC en los futuros maestros de educación infantil y primaria: hacia una alfabetización tecnológica real para docentes. Pixel-Bit. Revista de medios y Educación, 47, 23-39. doi: 10.12795/pixelbit.2015.i47.02

Royaume du Maroc (1999). Charte Nationale de l'Education et de la Formation (CNEF). Rabat. Recuperado de http:// www.men.gov.ma

Trujillo, J. M., Aznar, I. \& Cáceres, M. P. (2015). Análisis y uso e integración de redes sociales colaborativas en comunidades de aprendizaje de la Universidad de Granada y John Moores de Liverpool. Revista complutense de educación, 26 (1), 289-311.

UNESCO (2009). La Unesco y la educación. La educación: un derecho humano. París: UNESCO. Recuperado de http://unesdoc.unesco.org/images/0018/ 001849/184967s.pdf

UNESCO (2014). Informe de seguimiento de la EPT en el mundo 2013/14. Enseñanza y aprendizaje: Lograr la calidad para todos. Educación para todos. Paris: UNESCO. Recuperado de http://unesdoc.unesco.org/ images/0022/002261/226159s.pdf

UNESCO (2015). Educación 2030: Hacia una educación inclusiva y equitativa de calidad y un aprendizaje a lo largo de la vida para todos. Declaración de Incheon. Incheon: Foro Mundial sobre la Educación 2015. Recuperado de http://es.unesco.org/ world-education-forum-2015/about-forum/ declaracion-de-incheon

Valverde, J., Garrido, M. C. \& Fernández, M. R. (2010). Enseñar y aprender con tecnologías: un modelo teórico para las buenas prácticas educativas con TIC. Teoría de la educación: educación y cultura en la sociedad de la información, 11 (1), 203-229.
Fecha de recepción: 10-03-2016

Fecha de evaluación: 30-03-2016

Fecha de aceptación: 14-04-2016

$-63-$

Píxel-Bit. Revista de Medios y Educación. $N^{o} 50$ Enero 2017. ISSN: 1133-8482. e-ISSN: 2171-7966. doi: http://dx.doi.org/10.12795/pixelbit.2017.i50.03 